INRA Prod. Anim., 2008, $21(5), 427-440$

\section{La production laitière dans les régions de l'arc Atlantique européen}

\author{
V. CHATELLIER ${ }^{1}$, A.PFLIMLIN ${ }^{2}$, C. PERROT ${ }^{2}$ \\ ${ }^{1}$ INRA, UR1134 LERECO, F-44316 Nantes, France \\ 2 Institut de l'Elevage, 149 rue de Bercy, F-75595 Paris, France \\ Courriel :vchatel@nantes.inra.fr
}

Les exploitations laitières localisées dans les régions limitrophes de l'océan Atlantique, qui sont hétérogènes, contribuent au quart de la production laitière communautaire. Les systèmes laitiers du sud sont très intensifs à la vache et à la surface et fortement dépendants des achats d'aliments concentrés. Les systèmes de l'Ouest de la France sont assez économes en aliments, mais pénalisés par des frais élevés de mécanisation. Les système laitiers du Royaume-Uni et de l'Irlande sont herbagers, économes mais obtiennent un faible prix du lait.

Les exploitations laitières européennes se sont, au fil des dernières décennies, fortement restructurées dans un contexte caractérisé par un contingentement de la production laitière, une amélioration des performances animales et un développement soutenu des techniques agricoles. Cette restructuration n'a cependant pas conduit à une standardisation et/ou à une homogénéisation des modèles productifs au sein de l'Union européenne (UE). Ainsi, la diversité des exploitations laitières demeure toujours importante. Elle est influencée principalement par les conditions du milieu naturel (relief, climat, potentiel agronomique), l'histoire agraire du pays, la densité de population, les stratégies régionales de valorisation du lait (filières fromagères de qualité, produits industriels...) ou encore les règles de la Politique Agricole Commune (PAC).

Les exploitations laitières des différentes régions européennes s'inscrivent actuellement (en 2008) dans une conjoncture assez particulière qui renforce l'intérêt de conduire des analyses comparatives visant à mieux comprendre les principales forces et faiblesses des systèmes techniques privilégiés. Ainsi, la forte augmentation du prix du lait intervenue au premier semestre de l'année 2008 (d'environ un tiers par rapport à l'année antérieure) n'a pas eu le même effet économique bénéfique pour tous les systèmes techniques laitiers, car certains ont été affectés, de façon concomitante, par la hausse du prix des céréales, des aliments du bétail, du carburant et des engrais. La dernière réforme de la PAC a, par ailleurs, modifié le cadre économique dans lequel les exploitations laitières évoluent. Cette réforme s'est traduite par une modification des règles de l'intervention sur les marchés de produits laitiers ; l'augmentation modérée des quotas laitiers, avant leur probable disparition à l'horizon de 2015 ; l'instauration de paiements directs attribués à la tonne de quota ; le renforcement des règles de la conditionnalité des soutiens directs; l'introduction d'un découplage des mesures de soutien des revenus agricoles, avec une assez forte latitude laissée aux Etats membres dans les modalités pratiques de sa mise en œuvre (découplage partiel versus découplage total ; référence historique versus mutualisation du paiement unique) ; un renforcement des mesures du développement rural grâce à une modulation obligatoire des aides directes du premier pilier.

Dans ce cadre, cet article propose d'apporter un éclairage sur l'hétérogénéité d'une partie des exploitations laitières européennes, à savoir celles localisées dans les régions européennes jouxtant l'océan Atlantique. Plus précisément, il porte sur les régions de l'arc Atlantique ayant participé au projet de recherche "Green Dairy» (Pflimlin et al 2006). Ce projet avait pour objectif de comparer l'impact des systèmes lai- tiers sur l'environnement et de rechercher des voies à l'adoption de systèmes plus durables. Il concernait les régions suivantes : République d'Irlande ; Irlande du Nord; Ecosse ; Pays de Galles ; Ouest de l'Angleterre ; Bretagne ; Pays de la Loire ; Aquitaine ; Pays-Basque espagnol ; Galice ; Nord du Portugal (Entre Douro, Minho/Beira). Bien que situées dans la même zone bio-géographique tournée vers l'océan Atlantique, les exploitations de ces régions relèvent d'une forte diversité de milieux et de climats. Les étés sont chauds et secs au Sud justifiant l'irrigation alors qu'ils sont doux et relativement bien arrosés au Nord donc favorables à la prairie et au pâturage. La place de la production laitière dans la production agricole totale varie selon les régions en passant de moins de $10 \%$ en Aquitaine et en Ecosse, à plus de $30 \%$ en Bretagne, en Galice et en Irlande.

Cet article s'appuie, d'une part, sur une revue de la littérature relative aux systèmes laitiers rencontrés dans ces régions et, d'autre part, sur un traitement des dernières données individuelles disponibles (2005) du Réseau d'Information Comptable Agricole Européen (RICA) européen. Il s'articule autour de trois sections : la première présente les principaux éléments méthodologiques utiles à la compréhension des résultats; la seconde s'intéresse à la dynamique des structures, à la productivité des facteurs de produc- 
tion et au modèle technique privilégié. La troisième porte sur les coûts de production du lait et les résultats économiques. Les propos de conclusion visent à discuter des atouts et contraintes des systèmes étudiés par rapport aux principaux enjeux du futur.

\section{1 / L'outil statistique et la définition des exploitations laitières}

Ce travail est basé sur les données du RICA de l'exercice 2005, enquête réalisée chaque année depuis plus de trente ans, de façon harmonisée, dans un échantillon d'exploitations agricoles de tous les Etats membres de l'UE. Le RICA est un outil statistique construit pour être représentatif des exploitations agricoles professionnelles ${ }^{1}$, pour lesquelles il donne des informations détaillées sur la structure, les résultats économiques et la situation financière. Les unités professionnelles assurent la quasi intégralité de la production laitière européenne (plus de 99\%).

Une première clé de segmentation a été appliquée de manière à isoler les exploitations dites «laitières». Sont considérées comme telles ici, toutes les exploitations agricoles ayant plus de cinq vaches laitières. Cette définition permet de regrouper l'intégralité de la production laitière en un seul type et de prendre en considération des exploitations associant d'autres productions agricoles à l'activité laitière (Chatellier et Jacquerie 2004, Blogowski 2003). Le RICA européen (UE à 152) compte un échantillon de 13096 exploitations laitières (tableau 1). Pour chaque exploitation de cet échantillon, un coefficient de pondération est déterminé de façon à ce que cet outil soit le plus représentatif possible de la diversité des exploitations telle qu'elle est mise en évidence par le recensement agricole ou les enquêtes de structures. Après extrapolation, l'UE à 15 compte 423400 exploitations laitières, dont 89800 sont localisées dans les onze régions étudiées. Ces régions représentent $23 \%$ du quota laitier de l'UE à 15 et $19 \%$ de celui de l'UE à 27 .

Dans cet article, l'analyse ne porte que sur les exploitations laitières dites «spécialisées». Sont considérées comme telles, les exploitations dont la valeur de la production laitière représente plus de $60 \%$ de la valeur de la production agricole totale (aides directes incluses). Ce choix est justifié par le fait que la comparaison des coûts de production (charges par tonne de lait) implique de disposer d'exploitations assez homogènes en terme de spécialisation. Les 66700 exploitations laitières spécialisées de l'arc Atlantique produisent $80 \%$ du lait de la zone. Si ces exploitations jouent un rôle important dans les régions du Nord et du Sud de l'UE, elles sont proportionnellement moins représentées dans les trois régions françaises.

Parmi les exploitations laitières spécialisées, une segmentation supplémentaire est réalisée de manière à isoler les unités ayant une production annuelle de lait supérieure à $200000 \mathrm{~kg}$. Cette dernière catégorie (43 400 exploitations pour $70 \%$ du lait de la zone considérée) est encore plus homogène. Elle permet de s'affranchir, dans l'analyse, des petites structures. Non seulement l'effectif de ces dernières a fortement baissé au cours de la dernière décennie, mais bon nombre d'entre elles ne seront vraisemblablement pas pérennes à moyen terme, notamment en raison des exigences environnementales qui impliquent une mise aux normes des bâtiments d'élevage.

Si le RICA est un outil construit pour être représentatif des exploitations agricoles, il convient cependant de rester prudent dans l'analyse des résultats issus de cases typologiques comportant un faible nombre d'individus enquêtés.

Tableau 1. Le nombre d'exploitations laitières selon plusieurs définitions (en 2005).

\begin{tabular}{|l|c|c|c|c|c|c|c|c|c|}
\hline & \multicolumn{3}{|c|}{ Ensemble } & \multicolumn{3}{c|}{ Exploitations spécialisées } & \multicolumn{3}{c|}{$\begin{array}{c}\text { Exploitations spécialisées } \\
>200 \text { T }\end{array}$} \\
\cline { 2 - 10 } & Echantillon & Univers & \% du lait & Echantillon & Univers & \% du lait & Echantillon & Univers & \% du lait \\
\hline Irlande & 440 & 22300 & 100 & 354 & 18200 & 86 & 248 & 10700 & 68 \\
Irlande du Nord & 115 & 4100 & 100 & 104 & 3600 & 94 & 90 & 3000 & 89 \\
Ecosse & 62 & 1600 & 100 & 53 & 1300 & 87 & 50 & 1200 & 86 \\
Pays de Galles & 127 & 3000 & 100 & 111 & 2700 & 94 & 106 & 2300 & 91 \\
Angleterre (SW) & 145 & 4800 & 100 & 114 & 3900 & 88 & 109 & 3700 & 87 \\
Bretagne & 310 & 19000 & 100 & 150 & 11700 & 62 & 124 & 9200 & 55 \\
Pays de la Loire & 184 & 12600 & 100 & 85 & 7000 & 60 & 63 & 5000 & 51 \\
Aquitaine & 70 & 3700 & 100 & 40 & 2200 & 69 & 27 & 1600 & 60 \\
Pays Basque & 116 & 700 & 100 & 116 & 700 & 96 & 90 & 500 & 89 \\
Galice & 296 & 14300 & 100 & 256 & 11800 & 92 & 115 & 4800 & 63 \\
Portugal (Nord) & 198 & 3700 & 100 & 194 & 3600 & 100 & 86 & 1500 & 75 \\
\hline Total 11 régions & $\mathbf{2 0 6 3}$ & $\mathbf{8 9 8 0 0}$ & 100 & $\mathbf{1 5 7 7}$ & $\mathbf{6 6 7 0 0}$ & $\mathbf{8 0}$ & $\mathbf{1 1 0 8}$ & $\mathbf{4 3 4 0 0}$ & $\mathbf{7 0}$ \\
\hline Régions Nord & 889 & 35800 & 100 & 736 & 29600 & 89 & 603 & 20900 & 81 \\
Régions France & 564 & $\mathbf{3 5 3 0 0}$ & 100 & 275 & 21000 & 62 & 214 & 15800 & 54 \\
Régions Sud & 610 & 18800 & 100 & 566 & 16100 & 94 & 291 & 6700 & 68 \\
\hline UE-15 & $\mathbf{1 3 0 9 6}$ & $\mathbf{4 2 3 4 0 0}$ & $\mathbf{1 0 0}$ & $\mathbf{8 4 7 0}$ & $\mathbf{2 6 9 6 0 0}$ & $\mathbf{7 4}$ & $\mathbf{5 4 6 0}$ & $\mathbf{1 5 8 7 0 0}$ & $\mathbf{6 3}$ \\
\hline
\end{tabular}

Sources : RICA UE 2005, Commission européenne DG AGRI-G3 / Traitement Institut de I'Elevage et INRA SAE2 Nantes.

\footnotetext{
${ }^{1}$ Les exploitations sont considérées comme professionnelles dès lors qu'elles emploient plus de 0,75 Unité de travail agricole (UTA) ou que leur Marge Brute Standard (MBS) dépasse un seuil minimal, fixé par Etat membre. Ce seuil est, par exemple, de 2 UDE au Portugal, en Espagne et en Irlande, 8 UDE en France et en Irlande du Nord, 16 UDE en Angleterre.

$2 \mathrm{Si}$, dans cet article, il est fait référence à l'UE à 15, les données du RICA sont désormais disponibles pour les nouveaux Etats membres. L'entrée de ces pays renforce encore l'hétérogénéité du secteur laitier européen, avec la co-existence de très petites structures familiales en Pologne et en Roumanie et de grandes unités dans d'autres pays tels que la République Tchèque.
} 


\section{2 / La dynamique des struc- tures, la productivité et les systèmes d'alimentation}

Cette seconde section s'intéresse aux caractéristiques structurelles des exploitations laitières de l'arc Atlantique. Elle distingue, d'une part, le rythme de restructuration des exploitations et la productivité des facteurs de production (travail, cheptel et terre) et, d'autre part, le modèle technique adopté (assolement, intensification et système d'alimentation).

\section{1 / La restructuration des exploitations et la productivité des facteurs}

Dans le secteur laitier, les gains de productivité ont été particulièrement rapides au cours des deux dernières décennies (CNIEL 2007). A l'échelle de l'UE à 15 et pour la période s'échelonnant de 1995 à 2007, la diminution du nombre d'exploitations laitières a été de $6,5 \%$ par an. Ce recul de l'effectif est supérieur à celui observé dans d'autres spéculations agricoles telles que la viande bovine ou les céréales. D'après les statistiques de la Commission européenne, la baisse du nombre d'exploitations n'a pas été homogène selon les pays et, pour chacun d'eux, selon les régions.

En Espagne, pays où les exploitations étaient initialement de petite dimension, la restructuration a été très forte (-13\% par an, soit le niveau le plus élevé de tous les pays européens). Le nom-bre d'exploitations laitières espagnoles est, en effet, passé de 132300 en 1995 à seulement 28500 en 2007. Cette évolution a entraîné une modernisation des structures pérennes et un accroissement de leur taille. Elle explique aussi une part des difficultés rencontrées actuellement par ce pays pour produire l'intégralité de son quota laitier, ce malgré une situation globalement déficitaire. En Galice (Maseda et al 2004), région qui regroupe près du tiers du quota laitier espagnol, la taille des structures pourrait prochainement rejoindre les niveaux observés dans l'Ouest de la France et en Irlande. Cette évolution devrait se poursuivre sur la base d'unités familiales ayant un recours limité au travail salarié (Institut de l'Elevage 2001a). Au Pays Basque, le rattrapage a été très rapide : la taille des troupeaux s'est accrue au rythme de deux vaches par an ; le rendement laitier par vache a progressé de $220 \mathrm{~kg}$ par an contre, par exemple, $80 \mathrm{~kg}$ par an dans l'Ouest de la France.

Au Royaume-Uni, la baisse du nombre d'exploitations laitières a été de $7 \%$ par an sur la période considérée (de 41100 exploitations en 1995 à 18300 en 2007), avec un recul cependant plus intense en Angleterre et aux Pays de Galles qu'en Ecosse et en Irlande du Nord. La production britannique de lait relève encore pour les deux tiers de l'Angleterre. Néanmoins, une part de la production (environ 5\%) a cependant quitté l'Angleterre (zone où la production laitière est en concurrence avec les grandes cultures) pour rejoindre l'Irlande du Nord, où la production laitière a augmenté d'un tiers depuis 1995 (McCluggage 2005). Une augmentation de la production a également été constatée au Pays de Galles $(+6 \%)$ et en Ecosse $(+8 \%)$. Cette évolution a été possible en raison du mode de gestion interne des quotas laitiers (Institut de l'Elevage 2006, Barthélemy et al 2000, Boinon 2000). En effet, les droits à produire peuvent changer de propriétaire de manière marchande, avec ou sans le foncier, sans limitation géographique interne au pays. Depuis plusieurs années, le Royaume-Uni ne parvient pas à réaliser le quota laitier dont il bénéficie, avec une sous-réalisation qui représente environ 2 à $3 \%$ des volumes. Son marché des droits à produire est devenu peu dynamique au regard de la situation des pays du Nord (Danemark et aux Pays-Bas). De même, la valeur de transaction du quota est devenue faible. Cette situation, qui s'est encore amplifiée suite à l'introduction du découplage, s'inscrit dans un contexte national où le prix du lait payé au producteur est parmi les plus faibles de l'UE (avec l'Irlande) et où la valorisation du coproduit viande bovine fut régulièrement dégradée suite aux crises sanitaires successives.

En Irlande, pays où les exploitations laitières sont historiquement plus petites qu'au Royaume-Uni (Institut de l'Elevage 2001b), le taux annuel de restructuration a été de $6 \%$ par an depuis 95. Dans ce pays, où la production de lait est cinq fois supérieure à la demande domestique, le nombre d'exploitations laitières est passé de 48000 en 1995 à 21800 en 2007. D'après les estimations du ministère de l'agriculture irlandais, ce rythme de restructuration devrait se maintenir dans les années à venir. Il tient à trois facteurs cumulés : la bonne situation économique du pays et le faible taux de chômage ont un impact plutôt défavorable sur l'installation en agriculture ; certains exploitants hésitent à réaliser les investissements rendus nécessaires du fait du classement, en 2005, de l'ensemble du pays en zones vulnérables (au sens de la directive Nitrates); le prix du foncier est très élevé, ce qui incite, malgré des résistances culturelles historiques sur cette question, certains producteurs à vendre leurs terres.

En France, le taux de restructuration a été plus faible (4\% par an depuis 1995) que dans les autres pays européens (Ruas 2002). Ce taux a cependant été plus vif dans les zones à faible densité laitière et dans celles où la production laitière coexiste avec les productions végétales. Néanmoins, les quotas laitiers ont jusqu'alors été géographiquement peu mobiles sur le territoire national (Institut de l'Elevage 2005). En effet, le régime des quotas laitiers est encadré de manière stricte, par des règles administratives, pour éviter une éventuelle délocalisation de la production de lait et favoriser l'installation de jeunes éleveurs. Ainsi, les droits à produire ne sont pas marchands et l'attribution de ces droits se fait gratuitement dans le cadre de procédures décentralisées à l'échelle de chaque département (Daniel 2002). Par conséquent, les gains de productivité du travail (quota laitier par emploi) ne sont pas identiques entre les exploitations des différentes régions dans la mesure où les possibilités d'attribution de quotas diffèrent selon l'intensité de la restructuration interne. En outre, les soutiens spécifiques accordés aux zones de montagne, via les mesures du Programme Hexagonal de Développement Rural (PDRH), favorisent le maintien de l'activité laitière dans les zones défavorisées et participent ainsi à une limitation de la restructuration.

Pour les exploitations laitières spécialisées des régions étudiées, la production de lait est, en moyenne, de $338000 \mathrm{~kg}$ par an, soit un volume proche de la moyenne communautaire (UE à 15). Elle varie de $215200 \mathrm{~kg}$ en Galice à un peu plus de $800000 \mathrm{~kg}$ dans le Sud-Ouest de l'Angleterre et en Ecosse (tableau 2). Avec une production comprise entre 260000 et $300000 \mathrm{~kg}$, les régions françaises occupent une position intermédiaire. Les exploitations irlandaises $(256000 \mathrm{~kg})$ assurent un volume de production correspondant à la moitié de celui des unités de l'Irlande du Nord. En ne se focalisant que sur les seules exploitations de plus de $200000 \mathrm{~kg}$, c'est-à-dire en écartant le tiers des 
Tableau 2. La production de lait par exploitation, par UTA, par vache et par hectare de SFP (kg).

\begin{tabular}{|c|c|c|c|c|c|c|c|c|}
\hline & \multicolumn{3}{|c|}{ Exploitations laitières spécialisées } & \multicolumn{3}{c|}{ Exploitations laitières spécialisées $>200$ T } \\
\cline { 2 - 8 } & / Exploitation & / UTA & / Vache & / Ha SFP & / Exploitation & / UTA & / Vache & $/$ Ha SFP \\
\hline Irlande & 256000 & 167300 & 5300 & 5520 & 345700 & 205600 & 5500 & 6110 \\
Irlande du Nord & 500800 & 291400 & 6590 & 8010 & 570000 & 319100 & 6690 & 8400 \\
Ecosse & 847300 & 308000 & 6810 & 8120 & 891300 & 317100 & 6860 & 8360 \\
Pays de Galles & 720000 & 331800 & 6690 & 7960 & 817200 & 358800 & 6680 & 8210 \\
Angleterre (SW) & 825900 & 323800 & 7240 & 10070 & 861000 & 329000 & 7270 & 10140 \\
Bretagne & 285300 & 165000 & 6880 & 6530 & 320900 & 172700 & 7070 & 6760 \\
Pays de la Loire & 297300 & 157200 & 7140 & 5670 & 357900 & 169000 & 7480 & 6070 \\
Aquitaine & 264700 & 179400 & 6690 & 7090 & 322800 & 202400 & 6890 & 7760 \\
Pays Basque & 564600 & 258100 & 8800 & 19490 & 770300 & 316000 & 9030 & 22280 \\
Galice & 215200 & 143100 & 5970 & 10870 & 367100 & 203800 & 6420 & 13670 \\
Portugal (Nord) & 250000 & 108900 & 7590 & 25830 & 455200 & 161900 & 8440 & 33850 \\
\hline Total 11 régions & 338000 & 191600 & $\mathbf{6 4 4 0}$ & $\mathbf{7 4 7 0}$ & $\mathbf{4 5 1 1 0 0}$ & $\mathbf{2 2 8 3 0 0}$ & $\mathbf{6 3 9 0}$ & $\mathbf{8 0 1 0}$ \\
\hline Régions Nord & 428500 & 238200 & 6210 & 7190 & 552700 & 277300 & 6400 & 7800 \\
Régions France & 287200 & 163500 & 6950 & 6250 & 332800 & 173900 & 7180 & 6590 \\
Régions Sud & 237600 & 139000 & 6500 & 13260 & $\mathbf{4 1 3 9 0 0}$ & 200300 & 7090 & 16940 \\
\hline UE-15 & $\mathbf{3 4 3 7 0 0}$ & $\mathbf{1 8 7 9 0 0}$ & $\mathbf{6 8 5 0}$ & $\mathbf{7 7 7 0}$ & $\mathbf{4 9 8 0 0 0}$ & $\mathbf{2 3 6 4 0 0}$ & $\mathbf{7 1 7 0}$ & $\mathbf{8 7 5 0}$ \\
\hline
\end{tabular}

Sources : RICA UE 2005, Commission européenne DG AGRI-G3 / Traitement Institut de l'Elevage et INRA SAE2 Nantes.

exploitations spécialisées, la production moyenne est de $451100 \mathrm{~kg}$ par an. La hiérarchie entre les régions subsiste, mais le rattrapage est net dans le cas des exploitations de Galice et d'Irlande.

En rapportant la production de lait à l'Unité de Travail Agricole (UTA), les hiérarchies subsistent, mais les écarts se resserrent légèrement. La quantité de lait produite par emploi dans les exploitations laitières spécialisées est particulièrement élevée au Pays de Galles (331 $800 \mathrm{~kg}$ par UTA), dans le SudOuest de 1'Angleterre et en Ecosse. Elle est deux fois plus importante qu'en Bretagne ou en Pays de la Loire et trois fois plus qu'au Portugal. Si les disponibilités en main d'œuvre familiale sont assez proches entre les régions (entre 1,4 et 1,9 UTA familiale), les exploitations britanniques ont un recours plus fréquent au salariat; celui-ci représente $39 \%$ de la main d'œuvre totale dans le Sud-Ouest de 1'Angleterre, contre seulement $4 \%$ dans les régions françaises, où les sociétés des types GAEC (Groupement agricole d'Exploitation en Commun) et EARL (Exploitations Agricoles à Responsabilité Limitée) sont plus développées. Si les écarts de productivité du travail sont importants, il importe de pondérer l'analyse en tenant compte du fait que, malgré l'introduction d'un seuil de spécialisation identique entre les zones (cf. §1), le taux de spécialisation pour le lait des exploitations spécialisées françaises est inférieur à celui des unités spécialisées des autres régions étudiées (le lait représente environ $70 \%$ de la production agricole totale dans les régions françaises contre près de $85 \%$ dans les régions des autres pays).
Les exploitations laitières spécialisées des régions de l'arc Atlantique ont une production annuelle de lait de $6640 \mathrm{~kg}$ par vache et de $7470 \mathrm{~kg}$ par hectare de Surface Fourragère Principale (SFP). Ces niveaux de production, qui augmentent légèrement pour les unités ayant une production supérieure à $200000 \mathrm{~kg}$, sont inférieurs à ceux rencontrés dans les autres pays du Nord de l'UE (Danemark, Pays-Bas, Allemagne du Nord). En outre, ils varient dans des proportions importantes entre les régions étudiées en fonction des modèles techniques privilégiés.

\section{2 / Les modèles techniques et les systèmes d'alimentation}

Les exploitations laitières spécialisées des régions de l'arc Atlantique ont, en moyenne, une Superficie Agricole Utilisée (SAU) de 53 hectares, dont $85 \%$ de SFP. Les superficies de maïs fourrage représentent $13 \%$ de la SFP, le chargement est de 1,9 Unité de Gros Bétail (UGB) herbivore par hectare de SFP et les achats en aliments concentrés (hors auto-utilisation des aliments produits sur l'exploitation) s'élèvent à 255 euros par UGB herbivore et par an. $\mathrm{Si}$ ces caractéristiques sont assez proches de celles observées en moyenne communautaire, de fortes disparités existent entre les régions étudiées (tableau 3).

Dans les régions du Sud, les disponibilités en foncier agricole sont particulièrement limitées. Ainsi, la SAU moyenne des exploitations laitières spécialisées est de seulement 10 hectares au Portugal, 20 hectares en Galice et 29 hectares au Pays Basque. Dans ces régions, le parcellaire est souvent très morcelé ce qui rend le recours au pâturage difficile, y compris en Galice où les surfaces de prairies sont majoritaires dans l'assolement. Pour compenser cette rareté du foncier, les éleveurs achètent parfois du fourrage (foin de luzerne ou ensilage de maïs), mais surtout de grandes quantités de concentrés. Ainsi, les achats en aliments concentrés s'élèvent 560 euros par UGB herbivore et par an au Portugal et à 1020 euros au Pays-Basque, alors qu'ils sont compris entre 150 et 175 euros en Irlande et dans les deux régions françaises de l'Ouest. Cette utilisation massive de concentrés au Pays-Basque (plus de 3 tonnes par vache et par an) ne semble pas poser de problèmes sanitaires importants et permet l'obtention d'un rendement laitier très élevé. Avec une production de $8800 \mathrm{~kg}$ de lait par vache et par an, soit $3500 \mathrm{~kg}$ de plus qu'en Irlande, les performances laitières sont les plus élevées non seulement d'Espagne mais également de l'ensemble des régions européennes. Dans une conjoncture marquée par une forte hausse du prix des végétaux (céréales, soja...), cette dépendance des exploitations espagnoles à l'égard des concentrés achetés devient une limite économique forte au système privilégié. Dans les régions du Nord du Portugal, la production de fourrages par hectare est très importante. Elle peut atteindre 25 tonnes de matière sèche, grâce à l'irrigation des surfaces de maïs fourrage et à l'implantation de cultures dérobées de ray-grass. Au Pays Basque et en Galice, la production fourragère est cependant moins abondante car les surfaces sont exploitées essentiellement sous la forme d'ensilage d'herbe. 
Tableau 3. La surface agricole, l'intensification et le coût en aliments concentrés.

\begin{tabular}{|c|c|c|c|c|c|c|c|c|}
\hline & \multicolumn{4}{|c|}{ Exploitations laitières spécialisées } & \multicolumn{4}{|c|}{ Exploitations laitières spécialisées $>200 \mathrm{~T}$} \\
\hline & $\begin{array}{c}\text { SAU / } \\
\text { exploitation } \\
\text { (hectares) }\end{array}$ & $\begin{array}{c}\text { Maïs } \\
\text { fourrage / } \\
\text { SFP }(\%)\end{array}$ & \begin{tabular}{|l} 
UGB \\
herbivores \\
/ ha de SFP
\end{tabular} & \begin{tabular}{|c|} 
Frais \\
aliments / \\
UGB \\
herbivore $(€)$
\end{tabular} & $\begin{array}{c}\text { SAU / } \\
\text { exploitation } \\
\text { (hectares) }\end{array}$ & $\begin{array}{c}\text { Maïs } \\
\text { fourrage / } \\
\text { SFP }(\%)\end{array}$ & \begin{tabular}{|c|} 
UGB \\
herbivores \\
/ ha de SFP
\end{tabular} & $\begin{array}{c}\text { Frais } \\
\text { aliments / } \\
\text { UGB } \\
\text { herbivore }(€)\end{array}$ \\
\hline \begin{tabular}{|l|} 
Irlande \\
\end{tabular} & 47 & 1 & 1,9 & 151 & 58 & 1 & 2,0 & 150 \\
\hline Irlande du Nord & 64 & 0 & 2,0 & 292 & 69 & 0 & 2,1 & 305 \\
\hline Ecosse & 119 & 0 & 2,1 & 310 & 122 & 0 & 2,2 & 314 \\
\hline Pays de Galles & 94 & 1 & 2,1 & 259 & 104 & 2 & 2,1 & 266 \\
\hline Angleterre (SW) & 92 & 10 & 2,1 & 310 & 95 & 10 & 2,1 & 311 \\
\hline Bretagne & 63 & 35 & 1,4 & 166 & 69 & 35 & 1,5 & 167 \\
\hline Pays de la Loire & 74 & 27 & 1,4 & 176 & 86 & 29 & 1,4 & 189 \\
\hline Aquitaine & 58 & 44 & 1,4 & 325 & 67 & 49 & 1,5 & 342 \\
\hline Pays Basque & 29 & 2 & 3,0 & 1020 & 35 & 2 & 3,4 & 1060 \\
\hline Galice & 20 & 7 & 2,3 & 440 & 27 & 12 & 2,7 & 470 \\
\hline Portugal (Nord) & 10 & 90 & 4,9 & 564 & 14 & 94 & 5,9 & 622 \\
\hline Total 11 régions & 53 & 13 & 1,9 & 255 & 67 & 14 & 1,9 & 262 \\
\hline Régions Nord & 63 & 3 & 2,0 & 229 & 75 & 4 & 2,1 & 239 \\
\hline Régions France & 66 & 33 & 1,4 & 183 & 74 & 34 & 1,4 & 192 \\
\hline Régions Sud & 18 & 21 & 2,7 & 515 & 25 & 29 & 3,1 & 566 \\
\hline UE-15 & 56 & 15 & 1,8 & 306 & 73 & 17 & 1,9 & 319 \\
\hline
\end{tabular}

Sources : RICA UE 2005, Commission européenne DG AGRI-G3 / Traitement Institut de l'Elevage et INRA SAE2 Nantes.

Dans les régions françaises de l'arc Atlantique, les exploitations laitières bénéficient de plus grandes disponibilités en foncier (Chatellier et Jacquerie 2005), ce d'autant que le prix des terres y est plus bas que dans la plupart des autres régions européennes. En fonction du potentiel agronomique des sols, ce prix varie souvent entre 3000 et 8000 euros par hectare contre, par exemple, 20000 à 25000 euros par hectare en Irlande ou dans certaines petites régions agricoles anglaises. Ces disponibilités en surfaces permettent de développer des systèmes alimentaires relativement autonomes. Ainsi, une production de céréales auto-consommées vient souvent compléter la production de fourrages. Le système fourrager repose principalement sur le maïs fourrage pour les stocks d'hiver et les transitions, et sur l'herbe pâturée au printemps et en été lorsqu'il ne fait pas trop sec (Chenais et al 2001, Peyraud et Delaby 2005). Les prairies temporaires à base de ray-grass sont prédominantes et sont intégrées dans la rotation ce qui permet d'obtenir de bons rendements en maïs fourrage ou en blé. Grâce à ces deux fourrages de bonne qualité (maïs ensilé et herbe pâturée), les quantités de concentrés achetées sont généralement inférieures à une tonne par vache et par an.

Dans les régions du Nord, la SAU des exploitations laitières est souvent importante et elle est essentiellement consacrée aux productions fourragères. Compte tenu du climat et des types de parcellaires, le modèle alimentaire pri- vilégié repose surtout sur les prairies de longue durée. Elles permettent de recourir au pâturage (6 à 8 mois par an, voire davantage en Irlande du Sud) et de constituer des stocks d'ensilage. En Irlande, $85 \%$ des vêlages sont groupés en fin d'hiver et l'essentiel de la production laitière se fait pendant la période de pâturage, avec une utilisation limitée de concentrés. Dans les régions britanniques, les vêlages d'automne restent prédominants et nécessitent donc davantage de stock d'ensilage et de concentrés. Avec une fertilisation minérale de 200 à 250 unités d'azote par hectare et une consommation de 1,5 à 2 tonnes de concentrés par vache, les chargements sont souvent supérieurs à deux UGB herbivores par hectare de SFP.

Trois grandes zones peuvent donc être distinguées au sein de l'arc Atlantique concernant les modèles techniques laitiers et leur relation à l'environnement $: i$ ) les régions du Sud qui sont en évolution rapide vers des systèmes laitiers très intensifs à la vache et à la surface, mais dans un environnement peu intensif et où les forêts jouent un rôle déterminant dans l'occupation du territoire ; ii) les régions de l'Ouest français où malgré des systèmes laitiers relativement économes et autonomes, la qualité de l'eau reste dégradée, principalement du fait de la présence concomitante de l'élevage hors sol ; iii) les régions du Nord assez intensives, mais avec des systèmes herbagers pérennes ne présentant pas de risques immédiats pour la qualité de l'eau.

\section{3 / Les résultats écono- miques et les coûts de pro- duction du lait}

Dans un contexte économique caractérisé par une modification des règles de l'Organisation Commune du Marché $(\mathrm{OCM})$ du lait et des produits laitiers, une baisse des droits de douane, une suppression des restitutions et un changement dans les modalités d'octroi des paiements directs, la question de la compétitivité relative des exploitations et des bassins de production se pose avec davantage d'acuité. L'analyse comparative des résultats économiques est d'autant utile que les exploitations sont localisées dans un même cadre concurrentiel (IFCN 2004). Elles le seront d'ailleurs d'autant plus demain que le régime des quotas pourrait disparaître. Cette comparaison des résultats se révèle cependant être délicate dans la mesure où les agriculteurs européens ne s'inscrivent pas tous dans un même cadre économique. Ainsi, pour un même niveau de revenu, le pouvoir d'achat des agriculteurs n'est pas nécessairement le même d'un pays ou d'une région à l'autre. De même, les agriculteurs britanniques peuvent être sensibles à l'évolution de la parité monétaire entre la livre sterling et l'euro. Si les indicateurs utilisés ci-après sont déterminés selon une méthode identique, les résultats peuvent être influencés par des considérations propres à chaque pays relativement au mode d'attribution des fonds du second pilier 
de la PAC, aux normes retenues pour la durée des amortissements ou encore aux règles fiscales (taux d'imposition des bénéfices).

\section{1 / La production agricole, le revenu et la situation financière}

L'analyse comparée des résultats économiques entre régions concerne uniquement les exploitations laitières spécialisées de plus de $200000 \mathrm{~kg}$ de lait. Ce choix permet de limiter l'impact spécifique des petites exploitations laitières, lesquelles se caractérisent par une productivité du travail inférieure et des revenus plus faibles. La valeur de la production agricole (y compris les aides directes) par UTA varie, aux extrêmes, de 57000 euros au Portugal à 128100 euros au Pays de Galles. Elle dépend surtout de la production de lait par emploi et du prix du lait. Ce dernier varie de façon importante entre les régions en fonction surtout du type de valorisation des produits laitiers. Il est particulièrement faible dans les régions du nord (265 euros par tonne), où la moitié du lait est valorisé sous la forme de lait de consommation. C'est également le cas en Irlande (267 euros) où $80 \%$ de la production est exportée. Il est plus élevé dans les régions espagnoles (312 euros) que dans les régions françaises (290 euros). Ainsi, les écarts de productivité du travail mis en évidence précédemment entre les différentes régions sont plus faibles lorsque la productivité est exprimée en valeur et non pas en volume.

Le niveau moyen d'efficacité économique, mesuré par le ratio «Excédent brut d'exploitation $3 /$ Production agricole + aides directes», varie assez fortement entre les régions étudiées. Ces écarts s'expliquent par l'effet cumulé d'un ensemble de facteurs (Hallam 1995) : la spécialisation agricole, le prix du lait, le montant des aides directes et, surtout, la structure des charges. Il est plus faible dans les régions britanniques (environ 33\%) que dans l'Ouest français $(42 \%)$. En Galice, ce ratio atteint $61 \%$, en raison d'une très bonne maîtrise des charges, à l'exception de celles relatives aux achats d'aliments. Les exploitations anglaises sont pénalisées par l'existence de charges salariales élevées. Du fait de ces niveaux distincts d'efficacité, les écarts observés entre régions sont moins importants au niveau de l'indicateur «EBE par UTA familiale» qu'ils ne le sont pour l'indicateur «production agricole (y compris les aides directes) par UTA totale».

Le Résultat Courant Avant Impôt $(\mathrm{RCAI})^{4}$ par UTA familiale s'élève, en moyenne, à 30800 euros dans les exploitations de l'arc Atlantique, soit $6 \%$ de moins que la moyenne communautaire. Le revenu est proche de 40000 euros dans le Sud-Ouest de l'Angleterre et au Pays de Galles, régions favorisées par une forte productivité du travail et des investissements limités. Le RCAI par UTA familiale est également supérieur à la moyenne en Irlande en raison de 1'obtention d'une excellente efficacité économique. Il est nettement plus élevé que celui des unités françaises de l'Ouest qui disposent pourtant d'une productivité du travail (exprimée en valeur) équivalente. Une des explications à cet écart tient au fait que les investissements réalisés pour la modernisation des bâtiments d'élevage ont été, dans l'Ouest de la France, particulièrement soutenus au cours des dix dernières années. En Aquitaine, la faiblesse de l'efficacité économique et du revenu doit être considérée avec prudence car la taille de l'échantillon du RICA est limitée. En outre, l'amélioration de la conjoncture céréalière devrait profiter économiquement à ces exploitations, souvent un peu moins spécialisées car orientées vers la production de maïs grain. En Galice, et pour les exploitations de plus de $200000 \mathrm{~kg}$, le RCAI est élevé. La situation du revenu est cependant moins favorable pour les nombreuses exploitations de petite taille, singulièrement dans le contexte d'une augmentation rapide du prix des aliments concentrés.

Au niveau financier, la structure du bilan des exploitations diffère fortement selon les régions de l'arc Atlantique. Le montant total du capital inscrit au bilan s'élève, en moyenne, à 772900 euros pour la catégorie d'exploitations laitières considérée (contre un million d'euros en moyenne communautaire). $\mathrm{Au}$ Royaume-Uni, ce montant intègre une valeur pour le quota laitier, y compris lorsque celui-ci n'a pas été acquis par la voie marchande. Autrement dit, lors de la mise en œuvre des quotas laitiers, une valeur a été artificiellement créée. En France, où les quotas laitiers ne sont pas marchands, la valeur est nulle. En outre, les différentiels importants de prix du foncier interfèrent beaucoup sur l'estimation de la valeur du capital des exploitations ; le prix du foncier à l'hectare est, par exemple, huit fois plus élevé en Irlande que dans les régions de l'Ouest de la France, où, de surcroît, le taux de fermage est plus élevé. Ainsi, le montant des capitaux inscrits au bilan est, rapporté à la tonne de lait produite, près de quatre fois supérieur dans les exploitations Irlandaises que dans les unités de l'Ouest de la France. Le montant des dettes rapporté à la tonne de lait produite est, quant à lui, plus faible en Irlande (166 euros par tonne) qu'en Bretagne (416 euros par tonne). Ce constat indique surtout que les capitaux acquis dans les entreprises irlandaises ne l'ont pas été nécessairement par la voie de l'emprunt. En effet, ces capitaux sont souvent transférés gratuitement d'une génération à l'autre, ce qui signifie que la valeur d'acquisition des exploitations est souvent très inférieure à leur valeur patrimoniale. Cela rappelle aussi que les investissements sont plus soutenus dans les exploitations françaises, dont les bâtiments d'élevage ont été modernisés. Le taux d'endettement est donc fortement influencé par la valeur estimée des éléments de l'actif. Le montant des dettes par tonne de lait produite est huit fois plus élevé dans les régions de l'Ouest de la France que dans celles de l'Espagne, alors que ces dernières ont pourtant fait l'objet d'une restructuration à marche forcée depuis dix ans.

\section{2 / Le montant de la produc- tion agricole et des charges par tonne de lait}

Dans les exploitations laitières spécialisées de l'arc Atlantique ayant une production laitière supérieure à $200000 \mathrm{~kg}$ par an, la production agricole totale (aides directes incluses) s'élève, en moyenne, à 183000 euros. Elle provient pour $69 \%$ de la commercialisation du lait, $13 \%$ des aides directes (dont l'aide directe laitière) et $18 \%$ de la vente d'autres produits agricoles (comme la viande bovine issue du cheptel laitier mais aussi, parfois, les céréales). Ainsi, dans ces exploitations, la valeur de la production agricole totale représente, en moyenne, 446 euros par tonne de lait produite (tableau 4), dont 303 euros correspondent stricto sensu à la vente de lait.

\footnotetext{
$3 \mathrm{EBE}=$ Production de l'exercice - Consommations intermédiaires - Loyers et fermage - Assurances + Rabais, ristournes et remises - Impôts et taxes - Charges de personnel + Remboursement de TVA + Subventions d'exploitation + Indemnités d'assurance.

${ }^{4} \mathrm{RCAI}=\mathrm{EBE}-$ Dotations aux amortissements - Frais financiers + Transfert de charges + Autres produits de gestion courante + Produits financiers
} 
Tableau 4. Les résultats économiques dans les exploitations spécialisées de plus de 200 Tonnes (euros, \%).

\begin{tabular}{|c|c|c|c|c|c|c|c|}
\hline & $\begin{array}{l}\text { Prix du lait } \\
\text { / Tonne }\end{array}$ & $\begin{array}{l}\text { Production } \\
\text { + Aides } \\
\text { / UTA }\end{array}$ & $\begin{array}{c}\text { EBE / } \\
\text { Production } \\
+ \text { Aides (\%) }\end{array}$ & $\begin{array}{l}\text { EBE / } \\
\text { UTAF }\end{array}$ & $\begin{array}{l}\text { RCAI } \\
\text { / UTAF }\end{array}$ & $\begin{array}{c}\text { Montant } \\
\text { des capitaux }\end{array}$ & $\begin{array}{l}\text { Dettes } \\
\text { / Tonne de } \\
\text { lait }\end{array}$ \\
\hline Irlande & 267 & 84900 & 52 & 51600 & 36800 & 1270000 & 166 \\
\hline Irlande du Nord & 260 & 107900 & 40 & 46900 & 28700 & 963200 & 146 \\
\hline Ecosse & 266 & 120200 & 32 & 56300 & 31700 & 1750000 & 207 \\
\hline Pays de Galles & 261 & 128100 & 34 & 60800 & 39500 & 980600 & 259 \\
\hline Angleterre (SW) & 264 & 119100 & 33 & 66500 & 45900 & 1090000 & 256 \\
\hline Bretagne & 289 & 83100 & 42 & 36500 & 20200 & 301200 & 416 \\
\hline Pays de la Loire & 295 & 83700 & 42 & 36900 & 21400 & 318500 & 382 \\
\hline Aquitaine & 292 & 95800 & 31 & 30500 & 14100 & 274400 & 281 \\
\hline Pays Basque & 322 & 116500 & 36 & 49900 & 36000 & 491700 & 152 \\
\hline Galice & 311 & 80200 & 61 & 53100 & 48800 & 689200 & 28 \\
\hline Portugal (Nord) & 314 & 57000 & 35 & 26600 & 19100 & 237300 & 53 \\
\hline Total 11 régions & 278 & 92500 & 42 & 45900 & 30800 & 772900 & 234 \\
\hline Régions Nord & 265 & 104300 & 41 & 55000 & 37100 & 1190000 & 207 \\
\hline Régions France & 290 & 84600 & 41 & 36000 & 20000 & 304000 & 391 \\
\hline Régions Sud & 312 & 76000 & 52 & 45500 & 39700 & 576400 & 50 \\
\hline UE-15 & 303 & 105200 & 42 & 53500 & 32800 & 1000000 & 457 \\
\hline
\end{tabular}

Sources : RICA UE 2005, Commission européenne DG AGRI-G3 / Traitement Institut de l'Elevage et INRA SAE2 Nantes.

Ce montant varie fortement d'une région à l'autre en fonction principalement du prix du lait (tableau 5); de la valorisation commerciale des bovins (poids de carcasse et prix de vente des vaches de réforme, engraissement ou non des jeunes bovins, taux de mortalité des veaux nés...); des montants d'aides directes allouées aux exploitations (les systèmes avec des superficies de maïs fourrage sont, sur ce plan, plus favorisés que les systèmes herbagers) ; de l'importance d'éventuelles autres productions agricoles associées.

Parmi les régions considérées, la production agricole totale par tonne de lait est, au plus bas, en Irlande du Nord
(339 euros). Les exploitations de cette région cumulent un faible prix du lait (260 euros par tonne), une mauvaise valorisation commerciale en viande bovine, un montant limité des aides directes (en l'absence de céréales et de maïs fourrage) et une spécialisation quasi-exclusive en production laitière. A l'autre extrême, et pour des raisons inverses à celles avancées précédemment, ce montant atteint 496 euros en Pays de la Loire. D'une manière générale, ce montant est plus élevé dans les trois régions françaises (485 euros) que dans les régions du Sud (380 euros) ou celles du Nord (376 euros). Dans l'analyse comparée de la performance économique des élevages, il importe tout autant de tenir compte de ces écarts de valeur générée à la tonne de lait produite que de ceux relatifs aux coûts de production.

Dans les exploitations laitières spécialisées de l'arc Atlantique ayant une production laitière supérieure à $200000 \mathrm{~kg}$ par an, le montant total des charges s'élève, en moyenne, à 129100 euros, soit l'équivalent de 286 euros par tonne de lait produite. Ce montant est donc supérieur au prix de vente de la tonne de lait, mais inférieur à la valeur totale de la production agricole rapportée à la tonne de lait (laquelle intègre notamment les aides directes et la valeur de vente des bovins).

Tableau 5. Le montant de la production agricole et des charges par tonne de lait (euros) dans les exploitations laitières spécialisées produisant plus de 200000 kg de lait par an.

\begin{tabular}{|l|c|c|c|c|c|}
\hline & $\begin{array}{c}\text { Production } \\
\text { + Aides } \\
\text { / Tonne de lait }\end{array}$ & $\begin{array}{c}\text { Charges } \\
\text { totales } \\
\text { / Tonne de lait }\end{array}$ & $\begin{array}{c}\text { Charges } \\
\text { opérationnelles } \\
\text { / Tonne de lait }\end{array}$ & $\begin{array}{c}\text { Charges } \\
\text { de structure } \\
\text { / Tonne de lait }\end{array}$ & $\begin{array}{c}\text { Marge } \\
\text { / Totte } \\
\text { / Tonne de lait }\end{array}$ \\
\hline Irlande & 412 & 255 & 112 & 143 & 157 \\
Irlande du Nord & 339 & 251 & 122 & 130 & 88 \\
Ecosse & 379 & 304 & 145 & 160 & 75 \\
Pays de Galles & 357 & 273 & 123 & 150 & 84 \\
Angleterre (SW) & 363 & 272 & 122 & 150 & 91 \\
Bretagne & 482 & 365 & 97 & 268 & 117 \\
Pays de la Loire & 496 & 370 & 106 & 263 & 126 \\
Aquitaine & 472 & 405 & 152 & 94 & 67 \\
Pays Basque & 369 & 293 & 187 & 48 & 76 \\
Galice & 393 & 167 & 120 & 103 & 84 \\
Portugal (Nord) & 352 & 268 & 163 & 146 & 120 \\
\hline Total 11 régions & 406 & $\mathbf{2 8 6}$ & $\mathbf{1 1 9}$ & 265 & 114 \\
\hline Régions Nord & 376 & 267 & 121 & 67 & 172 \\
Régions France & 485 & 371 & 105 & $\mathbf{2 0 2}$ & 103 \\
Régions Sud & 380 & 208 & 139 & & \\
\hline UE-15 & 446 & $\mathbf{3 4 3}$ & 140 & & \\
\hline
\end{tabular}

Sources : RICA UE 2005, Commission européenne DG AGRI-G3 / Traitement Institut de l'Elevage et INRA SAE2 Nantes. 
Figure 1. Le montant des charges par tonne de lait dans les exploitations spécialisées de plus de $200 \mathrm{~T}$.

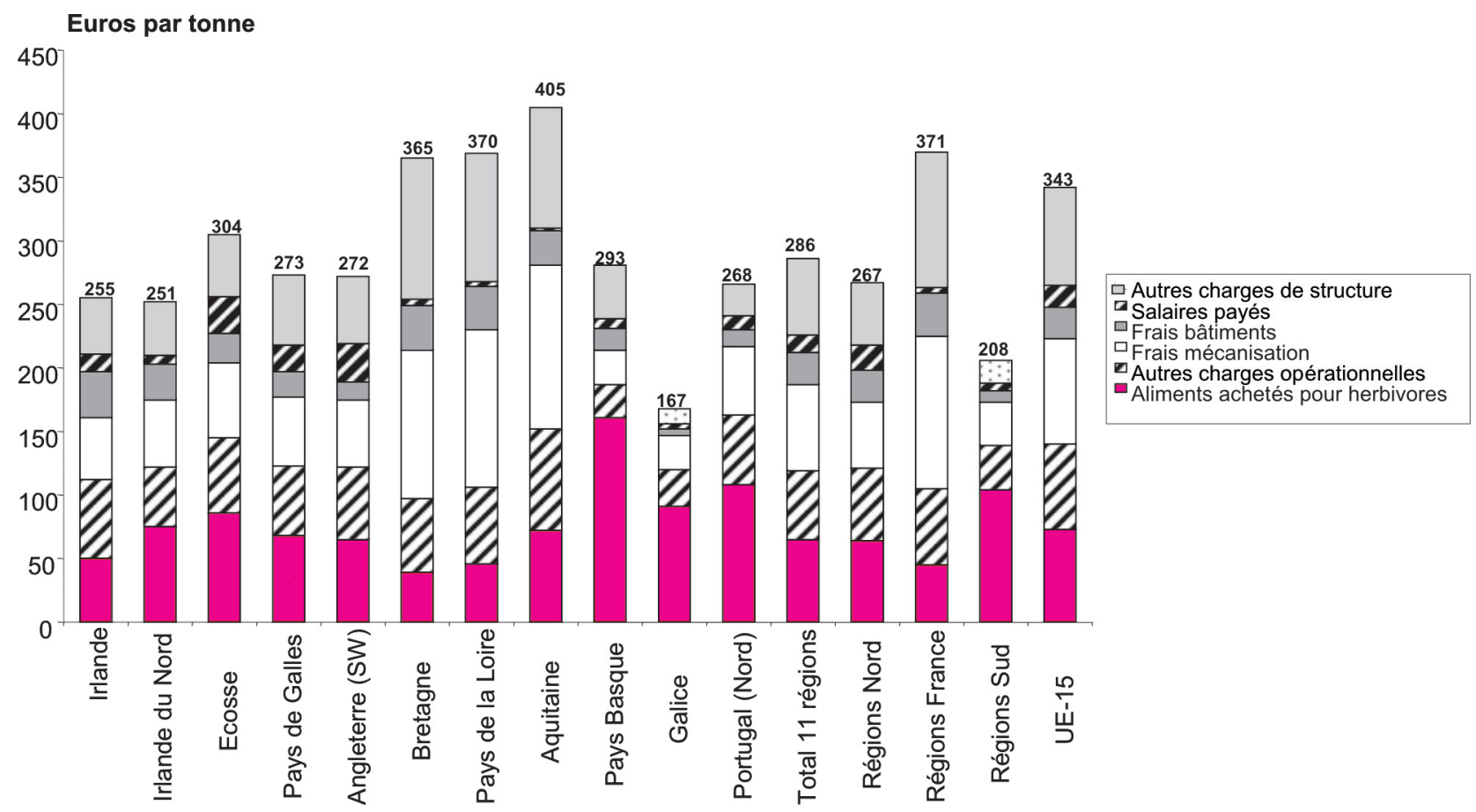

Sources : RICA UE 2005, Commission européenne DG AGRI-G3 / Traitement Institut de l'Elevage et INRA SAE2 Nantes.

Autrement dit, le revenu serait, en moyenne, négatif en l'absence des aides directes et des autres productions agricoles (viande bovine) associées à l'activité laitière. Dans le calcul, toutes les charges de l'exploitation sont prises en compte et non pas exclusivement celles liées à la production du lait. Sont également intégrées les charges relatives aux éventuelles autres productions (céréales, activité complémentaire de jeunes bovins) qui sont cependant, par définition, assez limitées dans la mesure où ces calculs sont réalisés pour les exploitations spécialisées. Les charges liées aux cultures peuvent être considérées comme relevant du secteur laitier dans la mesure où le produit de ces cultures est, pour une large part, intra-consommé sur l'exploitation par le cheptel laitier.

Le montant des charges par tonne de lait est plus faible dans la zone étudiée qu'en moyenne communautaire (- 57 euros par tonne). Cette meilleure maîtrise des coûts concerne aussi bien les charges opérationnelles (- 21 euros par tonne) que les charges de structure (- 36 euros par tonne). Néanmoins, cet atout (Saha 2003) est en partie contrebalancé par l'obtention d'un prix du lait inférieur à la moyenne communautaire (- 25 euros par tonne). Le prix du lait est, en effet, plus rémunérateur dans d'autres Etats membres de l'UE, tels que l'Autriche, le Danemark, l'Italie ou les Pays-Bas (Perrot et al 2007).
La marge nette, qui correspond à la différence entre la valeur de la production agricole et le montant des charges, s'élève en moyenne à 120 euros par tonne de lait dans les régions de l'arc Atlantique, soit 17 euros de plus que la moyenne communautaire. Cette marge nette est importante en Irlande (Thorne et Fingleton 2005). Elle semble l'être aussi en Galice (226 euros), mais des doutes sont cependant émis sur l'homogénéité du calcul des charges de structure (frais de mécanisation, frais de bâtiment, fermage, impôts et taxes, salaires payés, frais financiers) pour cette région par rapport aux autres Etats membres (le lecteur doit donc considérer avec prudence les résultats économiques de la Galice). Malgré un coût de production plus important, la marge par tonne de lait est plus élevée dans les régions de l'Ouest de la France (entre 115 et 130 euros) que dans les régions britanniques (entre 75 et 90 euros). Ce différentiel tient essentiellement aux écarts mis en évidence dans le prix du lait payé au producteur. Les exploitations britanniques sont néanmoins favorisées par une bien meilleure productivité du travail, qui permet l'obtention de revenus moyens supérieurs.

Le montant des charges est scindé en plusieurs rubriques de manière à identifier les forces et faiblesses des différentes régions. Avec 167 euros de charges par tonne de lait, les exploitations de
Galice sont les plus économes de l'arc Atlantique, ce malgré des frais assez élevés en alimentation du bétail (figure 1). Le montant des charges par tonne de lait est assez faible en Irlande du Nord (du fait d'un faible coût alimentaire) et en République d'Irlande (figure 1). Il est, en revanche, beaucoup plus élevé dans les régions françaises, principalement en raison du poids important des frais de mécanisation (environ 125 euros par tonne de lait contre moins de 30 euros au Pays-Basque et en Galice ou de l'ordre de 50 euros dans les régions britanniques). Les frais de mécanisation sont importants dans les régions françaises pour plusieurs raisons : les exploitations ne parviennent pas à diluer le poids des investissements en matériel grâce à un quota laitier élevé (comme, par exemple, dans le Sud-Ouest de l'Angleterre); elles ont des surfaces cultivables plus importantes que les autres, celles-ci étant destinées le plus souvent à l'alimentation du cheptel ; les investissements en matériel sont souvent privilégiés pour des raisons d'optimisations fiscales en cas d'obtention de résultats économiques satisfaisants. Moins dépendantes que les unités espagnoles des achats en aliments concentrés, les exploitations françaises sont favorisées par un coût alimentaire modéré à la tonne de lait. 
Figure 2. Le montant des charges par tonne de lait dans les exploitations laitières spécialisées, en fonction des quartiles de production de lait par UTA (euros, en 2005).

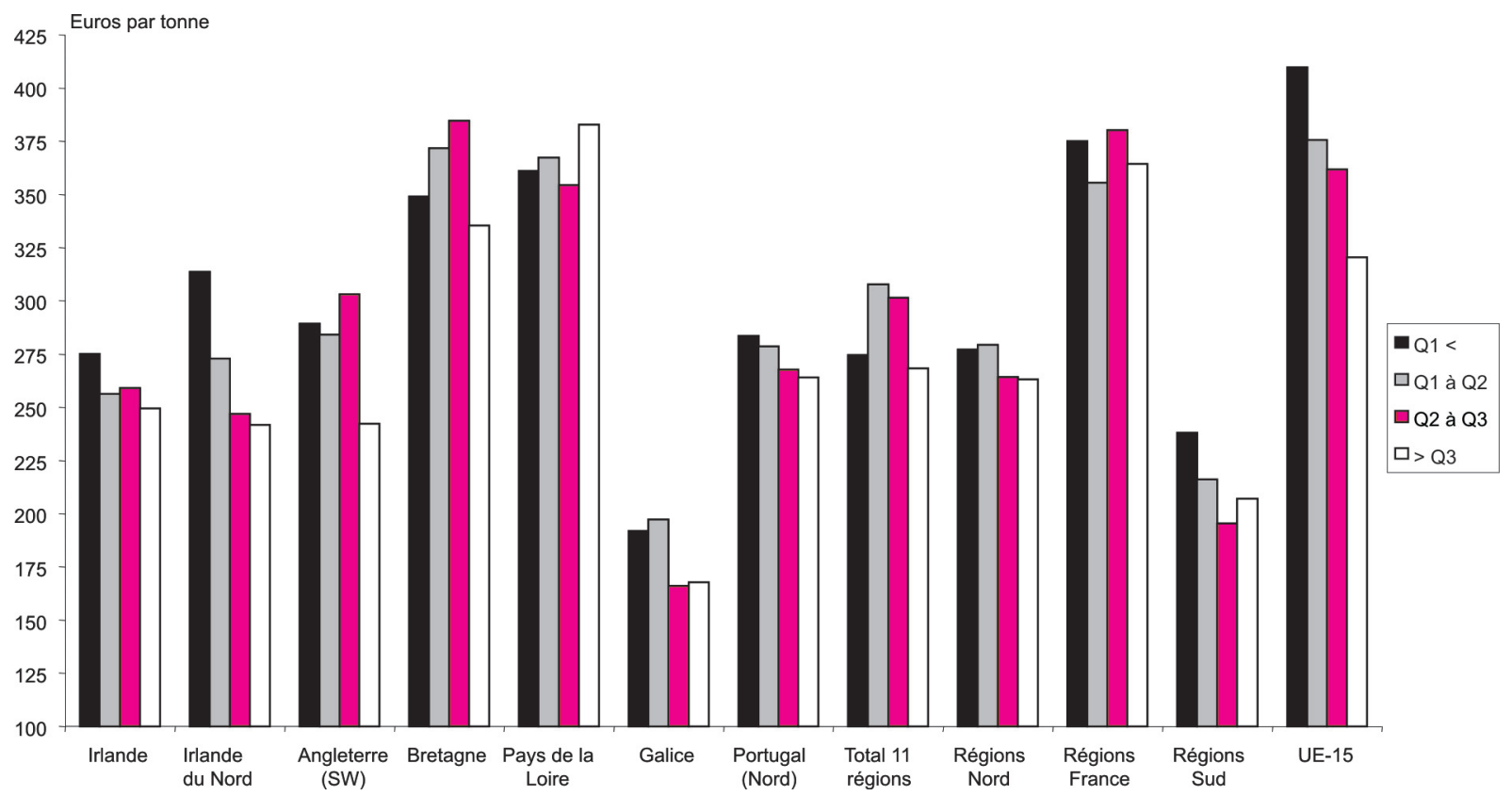

Sources : RICA UE 2005, Commission européenne DG AGRI-G3 / Traitement Institut de l'Elevage et INRA SAE2 Nantes.

\section{3 / La disparité intra-régiona-} le des résultats en fonction de la productivité du travail

La comparaison des résultats économiques entre régions européennes ne doit pas faire oublier l'existence de fortes disparités au sein de chacune d'elle. Pour mettre en évidence une partie de cette disparité, les exploitations laitières spécialisées (du moins pour les principales en termes d'effectifs) ont été réparties en quatre classes en utili- sant, pour chaque région, les valeurs quartiles de l'indicateur «production de lait par UTA».

Dans la grande majorité des régions, le montant des charges totales par tonne de lait diminue, mais seulement très légèrement, avec l'importance de la production de lait par emploi (figure 2). Cela suggère que les économies d'échelle (contraction des coûts de production en fonction de la taille) sont plutôt assez limitées $\mathrm{Au}$ niveau des charges de structure, les exploitations à plus haute productivité du travail sont pénalisées par une dynamique plus soutenue des investissements et donc par des dotations aux amortissements et des frais financiers plus élevés à la tonne de lait produite.

Dans toutes les régions étudiées, les exploitations bénéficiant de la meilleure productivité du travail sont aussi celles qui dégagent, en moyenne, le meilleur RCAI par UTAF (figure 3).

Figure 3. Le résultat courant avant impôt par UTA familiale dans les exploitations laitières spécialisées, en fonction des quartiles de production de lait par UTA (euros, en 2005).

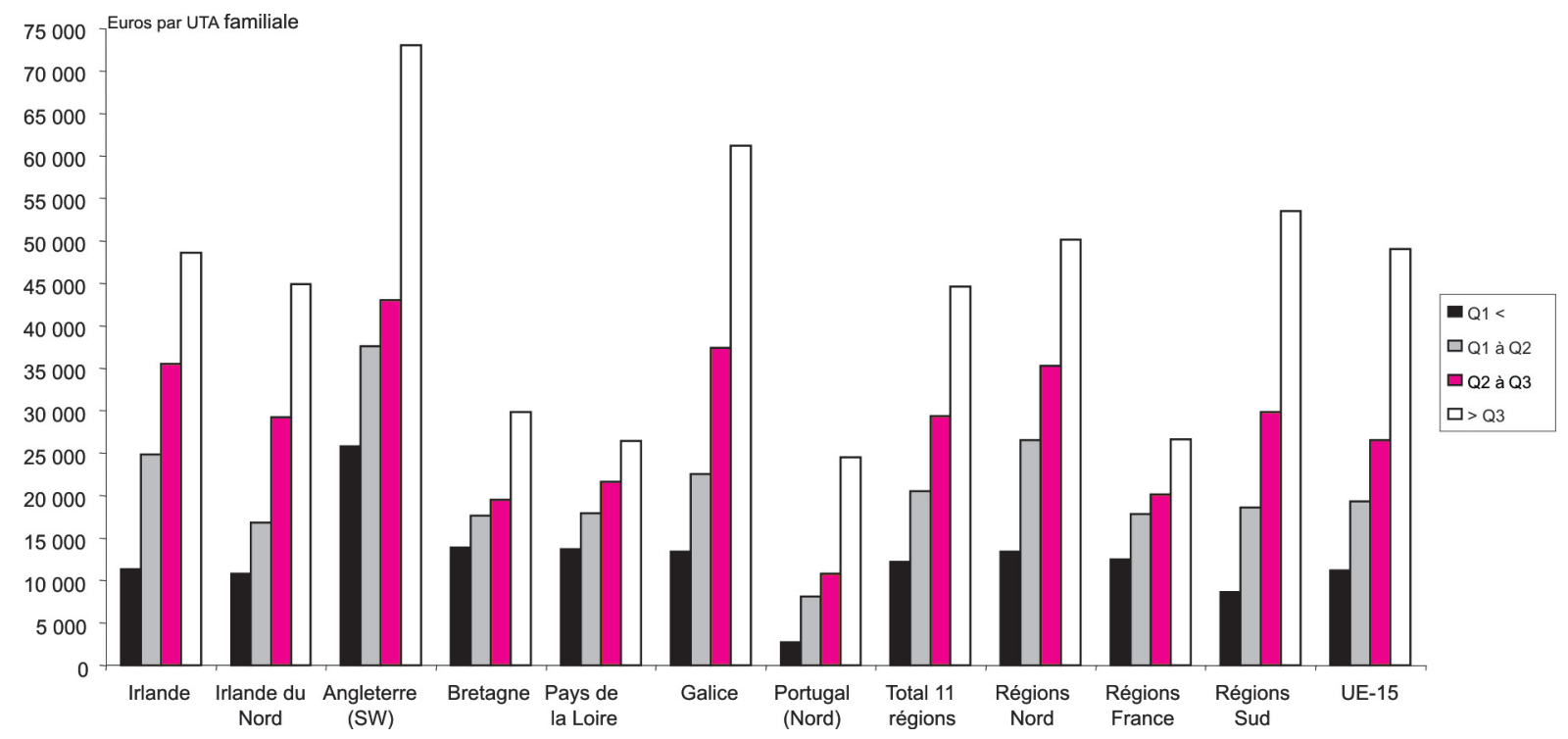

Sources : RICA UE 2005, Commission européenne DG AGRI-G3 / Traitement Institut de l'Elevage et INRA SAE2 Nantes. 
Cette relation tient beaucoup plus à l'effet du volume de production par emploi qu'à la marge nette dégagée par tonne de lait produite. L'effet de la productivité du travail sur le niveau de revenu est plus marqué en Irlande et dans les régions britanniques que dans les régions françaises. Cela s'explique essentiellement par le fait que les écarts de productivité entre les deux quartiles extrêmes sont plus accentués dans ces zones du nord où la politique agricole nationale est moins interventionniste. En France, le contrôle des structures conduit à limiter la taille des exploitations les plus grandes et donc à réduire les écarts entre les deux quartiles extrêmes.

Les exploitations ayant une haute productivité du travail sont, surtout en France, économiquement pénalisées par le fait qu'elles ont eu recours, souvent récemment, à des investissements importants pour moderniser leurs bâtiments. Au fil du temps et du remboursement des emprunts contractés, ces exploitations enregistreront une amélioration de leurs revenus. Pour les exploitations à faible niveau de productivité du travail, l'obtention d'un revenu rémunérateur passe souvent par une stratégie de limitation des investissements, laquelle pose immanquablement la question de la pérennité à long terme de l'outil de production.

\section{4 / Enjeux futurs et atouts et contraintes des bassins de production}

Les exploitations laitières situées dans les régions de l'arc Atlantique devront faire face, dans les années à venir, à plusieurs évolutions majeures, au même titre d'ailleurs que celles localisées dans les autres régions européennes. Sans faire de prédictions excessives, il semble que ces évolutions auront une influence importante sur les modèles techniques privilégiés et sur les choix stratégiques des agriculteurs. Les régions ne sont cependant pas interpellées de manière identique par toutes ces évolutions attendues qui fondent une partie des préoccupations actuelles des éleveurs.

\section{1 / Le renforcement des contraintes environnementales}

Face à la «Directive Nitrates», les exploitations laitières des régions françaises peuvent se prévaloir de disposer d'une avance non négligeable par rap- port à leurs homologues des pays du Nord et du Sud, notamment en ce qui concerne les capacités de stockage déjà réalisées et en cours d'amortissement (Le Gall et al 2005). La situation est différente dans les deux Irlande (Sud et Nord) qui ont déclaré, en 2005, toute l'île en zone vulnérable. De nombreux producteurs irlandais, pour lesquels la capacité de stockage du lisier est souvent inférieure à deux mois, réalisent désormais qu'ils doivent faire face à l'exigence d'investissements importants. Parmi les régions du Sud, seule la Galice pourrait accueillir davantage de lait car elle dispose d'importantes surfaces en herbe, exploitées de manière encore peu intensives. Cela supposerait, sans doute, un retour vers davantage de pâturage et une limitation des performances par vache. La «Directivecadre sur l'eau» présente, quant à elle, une plus grande inconnue. Il s'agit en effet d'obtenir, d'ici 2015, un bon état écologique de toutes les eaux (de surfaces, souterraines et côtières). Cet objectif conduira à mettre davantage en avant les problèmes d'eutrophisation qui interviennent à des concentrations en nitrates plus faibles que celles requises pour l'eau potable. De même, les seuils d'excédents de phosphore pourraient devenir plus limitant que ceux de l'azote.

\section{2 / Le renchérissement du prix de l'énergie et des intrants}

De nombreux prévisionnistes s'attendent à une augmentation du prix des énergies fossiles et, par voie de conséquence, du prix du carburant et des engrais. Par ailleurs, les organismes internationaux (FAO 2007, OCDE 2008) prévoient une hausse du prix international des produits agricoles (dont les céréales). Cette hausse des prix est potentiellement moins pénalisante pour les systèmes laitiers irlandais car ils sont particulièrement économes en aliments concentrés, en frais de mécanisation et en engrais minéraux. Les frais de fertilisation pourraient encore être réduits dans ce pays grâce au développement de la culture de trèfle blanc. Inversement, les systèmes laitiers des régions du Sud sont mal positionnés. Ils ont d'ailleurs été les plus durement affectés par l'augmentation du prix des céréales et des concentrés à partir de 2007. Ils pourraient être incités, dans un marché local $\mathrm{du}$ foncier cependant difficile, à rechercher des surfaces agricoles pour développer leurs productions fourragères et ainsi devenir progressivement plus autonomes. Dans les régions de l'Ouest de la France, une telle évolution est de nature à stimuler une réflexion collective sur les conditions à mettre en œuvre pour réduire les frais de mécanisation. Dans toutes les régions, la hausse du prix des intrants devrait inciter les agriculteurs à adopter progressivement des itinéraires techniques plus écologiques et, par voie de conséquence, plus respectueux de l'environnement (du moins dans les exploitations qui n'adoptent pas, déjà, cette stratégie).

\section{3 / Le découplage et les concur- rences entre productions agri- coles}

L'instauration du paiement unique constitue une évolution importante dans le mode d'intervention des pouvoirs publics en agriculture. Elle résulte de la volonté des autorités communautaires de rendre la PAC progressivement plus compatible avec les règles de l'Organisation Mondiale du Commerce (OMC). Suite à la réforme de la PAC de 2003, des aides directes ont été allouées sur la base du quota laitier. Elles ont ensuite été totalement découplées, ce de façon obligatoire dans tous les Etats membres. Ainsi, le montant du paiement unique dans les exploitations laitières correspond à un cumul entre les aides directes initialement allouées sur la base du quota laitier et d'éventuelles autres aides directes (aides aux superficies de grandes cultures, primes spéciales aux bovins mâles, primes à l'abattage...).

Pour les exploitations laitières diversifiées en grandes cultures et pour lesquelles la tentation existait d'abandonner la production laitière pour se consacrer uniquement à la production de céréales, l'instauration du découplage est, d'une certaine façon, une aubaine. En effet, ces exploitations peuvent désormais arrêter l'activité laitière tout en conservant le bénéficie des soutiens directs acquis au titre de cette activité. Dans une conjoncture où le prix des céréales est élevé, et compte tenu du travail astreignant qu'exige la production laitière, certains exploitants font ce choix. Ils sont cependant moins nombreux à le faire dans les régions étudiées de l'arc Atlantique car les exploitations ont des surfaces souvent limitées en grandes cultures. Le découplage total de la prime spéciale aux bovins mâles peut inciter à l'abandon de l'activité d'engraissement des jeunes bovins. Cette incitation dépend essentiellement des performances techniques de l'élevage, de la stratégie des groupements de producteurs et des fir- 
mes d'aval (par le développement de la contractualisation) et, surtout, du prix relatif entre les céréales et la viande bovine (Lelyon et al 2008). Dans les exploitations laitières de l'Ouest de la France, le découplage au taux de $75 \%$ des aides directes allouées aux superficies de maïs fourrage est un facteur qui pourrait théoriquement conduire à une relative extensification des systèmes laitiers. En effet, les exploitants peuvent conserver le bénéfice de ces primes tout en abandonnant cette culture au profit, par exemple, des prairies. Le choix des assolements dépend néanmoins de nombreux facteurs imbriqués tels que le potentiel agronomique des sols, les conditions climatiques, le mode d'organisation du travail sur l'exploitation ou le prix des céréales (un prix élevé stimule l'intensification des surfaces fourragères pour développer des cultures de vente). A ce stade, il est encore trop tôt pour tirer, à partir des observations statistiques disponibles, un bilan précis sur les implications productives du découplage.

Les règles appliquées par les Etats membres pour la mise en œuvre du découplage sont différentes (Bureau et al 2007). En France et en Espagne, il a été privilégié l'application d'un modèle historique, avec le découplage partiel de certaines primes (Chatellier 2006). En Irlande, le modèle historique a également été retenu, mais avec un découplage total. Au Royaume-Uni, il a été préféré le modèle hybride et dynamique à l'échelle de grandes régions. Les propositions faites par la Commission européenne le 20 mai 2008 au titre du bilan de santé de la PAC, suggèrent, d'une part, une réorientation partielle des aides directes à l'agriculture (par la modulation ou l'article 69 révisé du règlement $n^{\circ} 1782 / 2003$ ) et, d'autre part, une uniformisation progressive du montant du paiement unique par hectare entre les exploitations agricoles d'une même zone géographique (cette mesure restant facultative). Ces propositions suscitent de nombreux débats au sein des organisations professionnelles agricoles car elles posent la question de la justification future des soutiens publics accordés à l'agriculture. En France, la forte diversité des types de production et des systèmes techniques au sein d'une même région (systèmes intensifs à base de maïs fourrage versus systèmes extensifs à base d'herbe) renforce la complexité de ces débats. Dans d'autres régions, comme en Galice, où la surface moyenne par exploitation est modeste, et en Irlande, où les systèmes herbagers sont dominants, ces questions sont souvent considérées comme moins centrales par les pouvoirs publics et par les éleveurs..

\section{4 / La suppression des quotas laitiers}

La Commission européenne propose d'augmenter légèrement le volume du quota laitier d'ici 2015 pour parvenir à leur suppression à cette date. Elle indique que la suppression des quotas laitiers pourrait entraîner une baisse des prix, ce qui justifierait l'application, par les Etats membres, de mesures spécifiques dans les zones de montagne. La variation du prix du lait dépendra du marché international des produits laitiers, dans un contexte où, actuellement, l'offre peine à suivre la demande. Elle dépendra aussi de l'adéquation entre l'offre et la demande domestique de lait et donc de la stratégie des entreprises de transformation du lait, dont le rôle deviendra central en l'absence d'une régulation par la puissance publique. Sans spéculer donc sur ce que sera l'évolution réelle du prix du lait, force est de constater que la sensibilité des exploitations laitières à leur variation est loin d'être homogène. Ainsi, les exploitations du Sud-Ouest de l'Angleterre et de l'Ecosse sont parmi les plus sensibles. En effet, elles ont non seulement un volume de production important, mais une efficacité économique modeste. Ainsi, par exemple, une fluctuation de $20 \%$ du prix du lait entraîne une variation du revenu comprise entre $75 \%$ et $80 \%$ dans ces deux régions contre $30 \%$ en Galice, où les unités sont plus petites mais plus efficaces. Cette sensibilité est intermédiaire dans les régions de l'Ouest de la France.

La suppression des quotas laitiers pourrait conduire, en fonction surtout des stratégies adoptées par les entreprises de transformation du lait, à une concentration géographique accentuée de la production laitière. Au RoyaumeUni, le déplacement géographique de la production devrait se poursuivre dans les années à venir, avec ou sans le démantèlement des quotas laitiers. Pour autant, la croissance des volumes de production en Irlande du Nord deviendra plus modérée en raison des contraintes réglementaires qui s'appliquent dans le domaine de l'environnement. L'Irlande du Sud pourrait, quant à elle, accueillir plus de lait. En Espagne, la région de Galice a déjà bénéficié d'un transfert positif de quo- tas laitiers, mais cela a suscité certaines réactions politiques de la part des régions lésées. En France, la production de lait pourrait reculer dans les zones cumulant plusieurs handicaps : une faible densité de vaches laitières au $\mathrm{km}^{2}$; des exploitations individuelles de petite taille ; une valorisation commerciale modeste du lait. Les régions de l'Ouest de la France pourraient être confortées dans la mesure cependant où cette croissance des volumes demeure compatible avec les exigences environnementales, au niveau des petites régions agricoles ou des bassins versants.

\section{Conclusion}

En se focalisant sur une partie géographique limitée de l'UE, cet article a cherché à mettre en évidence la forte diversité des modèles de production adoptés dans le secteur laitier. Le climat, le potentiel agronomique des sols et les disponibilités en foncier agricole jouent et continueront de jouer un rôle déterminant dans l'orientation des systèmes laitiers. Dans toutes les régions de l'arc Atlantique, les exploitations laitières se sont fortement restructurées au cours des deux dernières décennies. Le rythme de restructuration a cependant été plus rapide dans les unités espagnoles, où l'intensification de la production est rendue nécessaire par la rareté du foncier. La hausse du prix des céréales et des aliments concentrés tend à rendre ces systèmes, pourtant économes en charges de structure, potentiellement fragiles. En Irlande, où la productivité du travail est modeste en comparaison de celle observée en Angleterre et en Irlande du Nord, le montant total du capital des exploitations est très élevé, mais les règles de transmission de celui-ci sont aussi assez spécifiques. Les normes environnementales qui imposent la modernisation des bâtiments d'élevage et la bonne situation économique du pays sont deux facteurs susceptibles de favoriser, dans les années à venir, une restructuration accélérée des exploitations. Comme leurs homologues du Royaume-Uni, les éleveurs irlandais sont pénalisés par l'obtention d'un faible prix du lait. Ils sont, en revanche, parmi les mieux positionnés au niveau du coût alimentaire. Les exploitations britanniques, dont les investissements en bâtiments et en matériels ont été peu soutenus depuis dix ans, parviennent à dégager des revenus par emploi supérieurs à la moyenne grâce à une très forte productivité. 
Les exploitations laitières de l'Ouest de la France ont connu, relativement aux autres régions, une augmentation moins rapide de leur quota laitier. Le montant des charges par tonne de lait est actuellement assez élevé, mais il pourrait diminuer dans les années futures grâce au remboursement progressif des emprunts contractés pour l'amélio- ration des bâtiments d'élevage et à une réduction des frais de mécanisation par tonne de lait (dans l'hypothèse d'une hausse des volumes de production). Si le montant des charges par tonne de lait est élevé, singulièrement pour les charges de structure, la valeur de la production agricole totale par tonne de lait produite l'est également. En effet, les éleveurs de l'Ouest de la France ont un prix du lait supérieur à celui des éleveurs anglais et irlandais, plus d'aides directes et une meilleure valorisation des productions associées au lait. Ils sont probablement plus sensibles que d'autres à l'évolution future des règles la PAC.

\section{Références}

Barthélémy D., Boinon J.P., Wavresky P, 2000. Droits à produire, des gestions nationales divergentes : l'exemple des quotas laitiers. INRA Sci. Sociales, 6, 4p.

Blogowski A., 2003. La diversité de l'agriculture européenne : les exploitations spécialisées en production laitière. Notes et Etudes Economiques, 18, 19-41.

Boinon J. P., 2000. La propriété des droits à produire. Econ. Rur., 260, 97-110.

Bureau J.C. et al, 2007. Reflection on the possibilities for the future development of the CAP. Final report for European Parliament Directorate General for Internal policies of the Union, $73 p$.

Chatellier V., 2006. Le découplage et les droits à paiement unique dans les exploitations laitières et bovins-viande en France. Cahiers Economie et Sociologie Rurales, 78, 53-80

Chatellier V., Jacquerie V., 2004. La diversité des exploitations laitières européennes et les effets différenciés de la réforme de la PAC. INRA Prod. Anim., 17, 315-333.

Chatellier V., Jacquerie V., 2005. L'occupation du territoire européen par les exploitations laitières et l'intensification de leurs systèmes techniques. Fourrages, 181, 29-45.

Chenais F., Seuret J.M., Brunschwig P., Fiorelli J.L., 2001. Pour un rôle croissant du pâturage dans les systèmes bovins laitiers. Fourrages, 166, 257-277.

CNIEL, 2007. L'économie laitière en chiffres, Paris, France, 217 p.

Commission européenne, 2007. Perspectives de marché dans le secteur du lait et des produits laitiers. Rapport au Conseil de la Commission européenne. Bruxelles, 20p.
Daniel K., 2002. Déterminants de la localisation des activités agricoles dans l'Union européenne : analyse des effets possibles de la suppression des quotas laitiers. Rapport INRA ADEPRINA, novembre, $182 \mathrm{p}$.

FAO, 2007. Food outlook. Report of the Food Agricultural Organisation, Rome, Italie, 64p.

Hallam M., 1995. Efficiency analysis with panel data: a study of Portuguese dairy farms. European Rev. Agric. Econ., 23, 5-93.

IFCN, 2004. Dairy Report, for better understanding of milk production world-wide, 152p.

Institut de 1'Elevage, 2001a. La filière laitière en Espagne : un développement sans gardefous. Dossier Economie Elevage, 304, 42p.

Institut de l'Elevage, 2001b. La filière laitière en Irlande : performante sans être novatrice. Dossier Economie Elevage, 306, 34p.

Institut de l'Elevage, 2005. Réforme de la PAC et production laitière : scénarios d'évolution à 1'horizon 2010-2012. Dossier Economie Elevage, 340, 72p.

Institut de l'Elevage, 2006. La filière laitière au Royaume-Uni, peu de valorisation pour la production. Dossier Economie Elevage, 361, 48p.

Le Gall A., Raison C., Bertrand S., Dockès A. C., Pflimlin A., 2005. Impact de la conditionnalité environnementale des aides de la PAC sur les systèmes laitiers français. Fourrages, 181, 67-95.

Lelyon B., Daniel K., Chatellier V., 2008. Decoupling and prices: determinant of dairy farmers choices? A model to analyse impacts of the 2003 CAP reform. 12 ${ }^{\text {th }}$ Congr. Eur. Assoc. Agric. Econom., EAAE, 26 august, 13p.

Maseda F., Diaz F., Alvarez C., 2004. Family dairy farms in Galicia, classification by some family and farm factors relevant to quality of life. Biosystems Engineering, 87, 509-521.

McCluggage I., 2005. Competitive dairying, the northern Ireland experience. National Dairy Conference 2005, Teagasc, Dublin, Irlande, 9199.

OCDE, 2008. Perspectives agricoles de l'OCDE et de la FAO 2008-2017. Synthèse de l'Organisation de la Coopération et du Développement Economique, Paris, France, 82p.

Perrot C., Chatellier V., Coulomb C., You G. 2007. Productivité et rémunération du travail dans les exploitations laitières du Nord de l'UE. Dossier Economie Elevage, 364, 62p.

Peyraud J. L., Delaby L., 2005. Combiner la gestion optimale du pâturage et les performances des vaches laitières : enjeux et outils. INRA Prod. Anim., 15, 231-240.

Pflimlin A., Raison C., Le Gall A., Irle A., Mirabal Y., 2006. Contribution du troupeau laitier aux excédents de $\mathrm{N}$ et $\mathrm{P}$ dans les régions Green Dairy. Renc. Rech. Rum., Paris, France, $4 \mathrm{p}$.

Ruas J. F., 2002. La réforme de la PAC de 1992 : bilan d'une décennie d'adaptation des élevages laitiers. Notes et Etudes Economiques, 16, 119-142.

Saha A., 2003. Method approach, cost of production versus technical efficiency. IFCN Dairy Report 2003, International Farm Comparison Network, Braunschweig,102-103.

Thorne F., Fingleton B., 2005. Irish dairy farming, can we compete ? National Dairy Conference 2005, Teagasc, Dublin, Irlande, 1025.

\section{Résumé}

Les régions de «l'arc Atlantique» qui participent au projet «Green Dairy» réalisent près du quart de la production laitière de l'UE. Au cours des deux dernières décennies, le taux de restructuration du secteur productif laitier a été particulièrement intense dans les régions espagnoles et anglaises. Dans les régions françaises, les mesures en faveur de l'installation de jeunes agriculteurs et la gestion administrée des quotas laitiers ont freiné le mouvement d'agrandissement des exploitations. Bien que situées dans la même zone climatique (sous influence océanique), les exploitations laitières de l'arc Atlantique privilégient des systèmes alimentaires variés et une utilisation plus ou moins intensive des surfaces fourragères. Du fait de surfaces limitées, les exploitations portugaises et espagnoles achètent d'importantes quantités de concentrés et sont souvent intensives. Les exploitations de l'Ouest de la France, qui valorisent fréquemment des fourrages ensilés, sont confrontées à des difficultés environnementales importantes, en raison de la présence d'autres productions animales (porcs et volailles). Favorisées par un faible coût alimentaire, elles sont pénalisées par des coûts de mécanisation élevés. Les unités irlandaises et anglaises, qui laissent une large place à l'herbe, ont un coût de production du lait bien maîtrisé. Elles sont cependant pénalisées par un 
faible prix du lait et investissent peu. Les exploitations laitières de l'arc Atlantique ne sont donc pas toutes homogènes face aux nouveaux défis : la remise en cause du régime des quotas laitiers (qui soulève d'importants enjeux territoriaux en France); le renchérissement du prix de l'énergie fossile (qui pourrait entraîner une hausse du prix des céréales, peu favorable aux systèmes alimentaires du sud) ; le renforcement des contraintes environnementales (qui implique des investissements).

\begin{abstract}
Dairy production in the European Atlantic Arc

The regions of the «Atlantic arc», which are involved in the «Green Dairy» project, realize nearly the quarter of European dairy production. During the last two decades, the decrease of the number of dairy farms was particularly intense in the Spanish and English areas. In France, the enlargement of farms was less important due to public choices (measurements in favour of young farmers and management of milk quotas). Although the dairy farms of the «Atlantic arc» are located in the same climatic zone (under oceanic influence), they privilege varied food systems and a more or less intensive use of fodder surfaces. Because of limited surfaces, the Portuguese and Spanish farms buy important quantities of animal food and are often intensive. The farms of the west of France, which frequently develop ensiled fodder, face important environmental problems, because this region produces many animal productions (pigs and poultry). Food cost is low but they are penalized by high costs of mechanization. The Irish and English units, for which food is provided mainly by grass, have a low production cost. However, they are penalized by a weak milk price and they invest little. The dairy farms of the «Atlantic arc» are thus not all equal when facing the new challenges: the future of the milk quota system (which raises important territorial stakes in France); the increase in the price of fossil energy (which could involve a rise in the price of cereals, unfavorable to the food systems of the south); the reinforcement of environmental constraints (which implies investments).
\end{abstract}

CHATELLIER V., PFLIMLIN A., PERROT C., 2008. La production laitière dans les régions de l'arc Atlantique européen. INRA Prod. Anim., 21, 427-440. 
